\title{
WestVirginiaUniversity
}

THE RESEARCH REPOSITORY @ WVU

West Virginia Agricultural and Forestry Experiment

Davis College of Agriculture, Natural Resources

Station Bulletins

And Design

$1-1-1969$

\section{Fortran IV program for stand table projection of forest growth}

Bryce E. Schlaegel

D. L. Kulow

Follow this and additional works at: https://researchrepository.wvu.edu/ wv_agricultural_and_forestry_experiment_station_bulletins

\section{Digital Commons Citation}

Schlaegel, Bryce E. and Kulow, D. L., "Fortran IV program for stand table projection of forest growth" (1969). West Virginia Agricultural and Forestry Experiment Station Bulletins. 583T.

https://researchrepository.wvu.edu/wv_agricultural_and_forestry_experiment_station_bulletins/689 @ WVU. It has been accepted for inclusion in West Virginia Agricultural and Forestry Experiment Station Bulletins by an authorized administrator of

The Research Repository @ WVU. For more information, please contact ian.harmon@mail.wvu.edu. 
West Virginia University Libraries 
Digitized by the Internet Archive in 2010 with funding from

Lyrasis Members and Sloan Foundation 


\section{FORTRAN IV PROGRAM \\ FOR STAND TABLE PROJECTION OF FOREST GROWTH}

Bulletin 583T

June 1969

West Virginia University Agricultural Experiment Station 


\section{THE AUTHORS}

Bryce Schlaegel was a Graduate Research Assistant at the time of this study; D. L. Kulow is Associate Forester.

\section{ACKNOWLEDGMENTS}

This program is one of the techniques used in obtaining growth and yield tables for the McIntire-Stennis Project Five, entitled "Growth and Yield of West Virginia Hardwoods."

The authors express thanks to the West Virginia University Computer Center for the use of its facilities in the preparation of the programs.

DISCLAIMER: Although this program has been tested and used, no warranty, expressed or implied, is made by the authors as to the accuracy and functioning of the program and related program material, nor shall the fact of publication constitute any such warranty, and no responsibility is assumed by the authors in connection therewith.

West Virginia University Agricultural Experiment Station College of Agriculture and Forestry

A. H. VanLandingham, Director

Morgantown 


\section{FORTRAN IV PROGRAM \\ FOR STAND TABLE PROJECTION OF FOREST GROWTH}

\section{Bryce Schlaegel and D. L. Kulow}

In 1964, a McIntire-Stennis study on the growth and yield of West Virginia hardwoods was initiated. The first data to be analysed were from 123 permanent sample plots in yellow-poplar (Liriodendron tulipifera, L.) and required the projection of basal area and stand volume for growth prediction. The method selected was one used by Ineson $(1947)^{\prime}$ called the stand projection method, where measurements of diameter and the length of the last ten years of radial growth are required. When a large number of observations are available, the procedure becomes tedious; so it can best be handled with an electronic computer. This bulletin describes a program written for the IBM $7040 / 7044$ (16/32K Operating System) using FORTRAN IV. It will handle up to 9,998 plots with 3,000 observatons on each plot. The range of tree diameters is from five to 35 inches, and diameter growth may be from zero to five inches in ten years.

\section{PROCEDURE}

Data required for the program are average volume per tree diameter (local volume table), diameter breast high to the nearest one-tenth inch, and the length of the last ten years of radial growth to the nearest one-tenth inch. Input is by punched cards, while output is on paper and on a plot basis. Table 1 is a listing of the FORTRAN IV program and Table 2 is a definition of the variables. Figure 1 shows the flow chart.

As the data are entered into the computer the trees are sorted into one-inch diameter classes from five inches up to a maximum of 35 inches. This represents the present stand table for each plot. At the same time, the trees are sorted into one-

'Ineson, F. 1947. Description of the stand projection method. U.S.F.S., Northeast Forest Survey. Second techniques meeting. 
tenth inch growth classes of zero to five inches. The per cent of the present stand table that occurs in each growth class is computed, as well as the number of trees that are expected to change one, two, three, etc., growth classes in the next ten years. The summation of these changing trees will give the future stand table.

The volumes for the present and future stock table are found by entering the average volume per diameter class (Table 3 ) into the computer and applying these to the respective stand tables. Volume growth, per plot, is the difference between the two stock tables. All values are then converted to the acre basis by use of the appropriate expansion factor.

Present and future basal area is found in the same manner. The square of the mid points of each diameter class are multiplied by 0.005454 and multiplied by the respective number of trees in each stand table. The difference between the two basal area columns is basal area growth. 


\section{DIMENSION VOL(36)}

DIMENSION DIA(3000), GRO(3000), ND(3000), NGRNO $(40,7), \operatorname{CLP}(40,7), \operatorname{MDIA}($ 140), NTMU $(40,7), \operatorname{NFST}(40), \operatorname{NPST}(50), \operatorname{NPSTK}(50), \operatorname{NFSTK}(50)$

4000 FORMAT( $1 \mathrm{H} 1,9 \mathrm{X}, 73 \mathrm{H} *$ PRESENT* NUMBER OF TREES MOVING UP PER GROW ITH CLASS * FUTURE *)

4001 FORMAT $(1 \mathrm{H}, 9 \mathrm{X}, 10 \mathrm{H} *$ STAND *,53X,10H* STAND *)

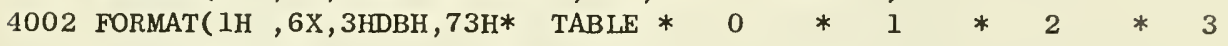

$1 * 4 * 5 *$ TABLE *)

4003 FORMAT( $1 \mathrm{H}, 6 \mathrm{X}, 19(4 \mathrm{H} * * * *))$

4004 FORMAT( $1 \mathrm{H}, 6 \mathrm{X}, \mathrm{I} 3,1 \mathrm{H} *, 8(\mathrm{I} 8,1 \mathrm{H} *)$ )

4005 FORMAT $(1 \mathrm{H}, 10 \mathrm{X}, \mathrm{I} 8,55 \mathrm{X}, \mathrm{I} 8)$

5100 FORMAT (I4, I4, F5.1, F4.1)

5302 FORMAT $(\mathrm{F} 6.2)$

5303 FORMAT $(14,12)$

5992 FORMAT( $1 \mathrm{H}, 5 \mathrm{X}, \mathrm{I} 3,1 \mathrm{H} *, 16,1 \mathrm{H} *, 6(\mathrm{I} 5, \mathrm{~F} 6.1,1 \mathrm{X}, 1 \mathrm{H} *))$

5993 FORMAT ( $1 \mathrm{H}, 5 \mathrm{X}, 22(4 \mathrm{H} * * * *), 1 \mathrm{H} *$ )

5994 FORMAT $(1 \mathrm{H}, 4 \mathrm{X}, 12 \mathrm{HDIA} *$ TREES*,6(13H NUM。 PCENT *))

5995 FORMAT ( $1 \mathrm{H}, 8 \mathrm{X}, 8 \mathrm{H} * \mathrm{NO}$ 。 OF*, 6(12X,1H*))

5996 FORMAT( $1 \mathrm{H}, 8 \mathrm{X}, 1 \mathrm{H} *, 6 \mathrm{X}, 1 \mathrm{H} *, 5 \mathrm{X}, 1 \mathrm{H} 0,6 \mathrm{X}, 1 \mathrm{H} *, 5 \mathrm{X}, 1 \mathrm{H} 1,6 \mathrm{X}, 1 \mathrm{H} *, 5 \mathrm{X}, 1 \mathrm{H} 2,6 \mathrm{X}, 1 \mathrm{H} *$ $1,5 \mathrm{X}, 1 \mathrm{H} 3,6 \mathrm{X}, 1 \mathrm{H} *, 5 \mathrm{X}, 1 \mathrm{H} 4,6 \mathrm{X}, 1 \mathrm{H} *, 5 \mathrm{X}, 1 \mathrm{H} 5,6 \mathrm{X}, 1 \mathrm{H} *)$

5997 FORMAT ( $1 \mathrm{H}, 15 \mathrm{X}, 26$ (3H***), 1H*)

5998 FORMAT(/////16X,1H*, 26X, 25HCLASSES MOVED IN 10 YEARS, 26X, 1H*)

6000 FORMAT $(1 \mathrm{H} 1,12 \mathrm{X}, 11 \mathrm{H} *$ PRESENT *,16H PRESENT *,16H FUTURE

$1 *, 16 \mathrm{H} \quad$ FUTURE *)

6001 FORMAT $(13 \mathrm{X}, 11 \mathrm{H} *$ STAND $*, 16 \mathrm{H}$ STOCK $*, 16 \mathrm{H}$ STAND *,

\section{$116 \mathrm{H}$ STOCK *)}

6002 FORMAT( $10 \mathrm{X}, 14 \mathrm{HDBH} *$ TABLE *,3(16H TABLE *))

6003 FORMAT $(10 \mathrm{X}, 31(2 \mathrm{H} * *))$

6004 FORMAT( $1 \mathrm{H}, 9 \mathrm{X}, \mathrm{I3}, 1 \mathrm{H} *, \mathrm{I9}, 1 \mathrm{H} *, 3(\mathrm{I} 15,1 \mathrm{H} *))$

6005 FORMAT( $1 \mathrm{HO}, 24 \mathrm{X}, \mathrm{I} 15,17 \mathrm{X}, \mathrm{I} 15)$

6006 FORMAT (1HO, 10X, 17HTOTAL GROWTH $=$, I15)

6007 FORMAT ( 1 HO , 1OX, 17HGROWTH PERCENT $=$, F9.4)

6008 FORMAT $(1 \mathrm{H} 1,10 \mathrm{X}, 37$ HTHE FOLLOWING DATA IS FOR PLOT NURBER, I5)

6009 FORMAT( 1 HO, 10X, 17HGROWTH PER ACRE $=, I 15)$

6010 FORMAT (1H0, 10X, 21HPRESENT BASAL AREA = ,F9.4)

6011 FORMAT $(1 \mathrm{HO}, 10 \mathrm{X}, 20$ HFUTURE BASAL AREA $=$,F9.4)

6012 FORMAT $(1 \mathrm{HO}, 10 \mathrm{X}, 29 \mathrm{HBASAL}$ AREA GROWTH PER ACRE $=$, F9.4)

6013 FORMAT( I2)

DO $1 \quad I=1,36$

$1 \operatorname{VOL}(I)=0$.

$\operatorname{READ}(5,6013) \mathrm{NV}$

DO $3 \quad I=I, N V$

$3 \operatorname{READ}(5,5302) \operatorname{VOL}(\mathrm{I})$ 


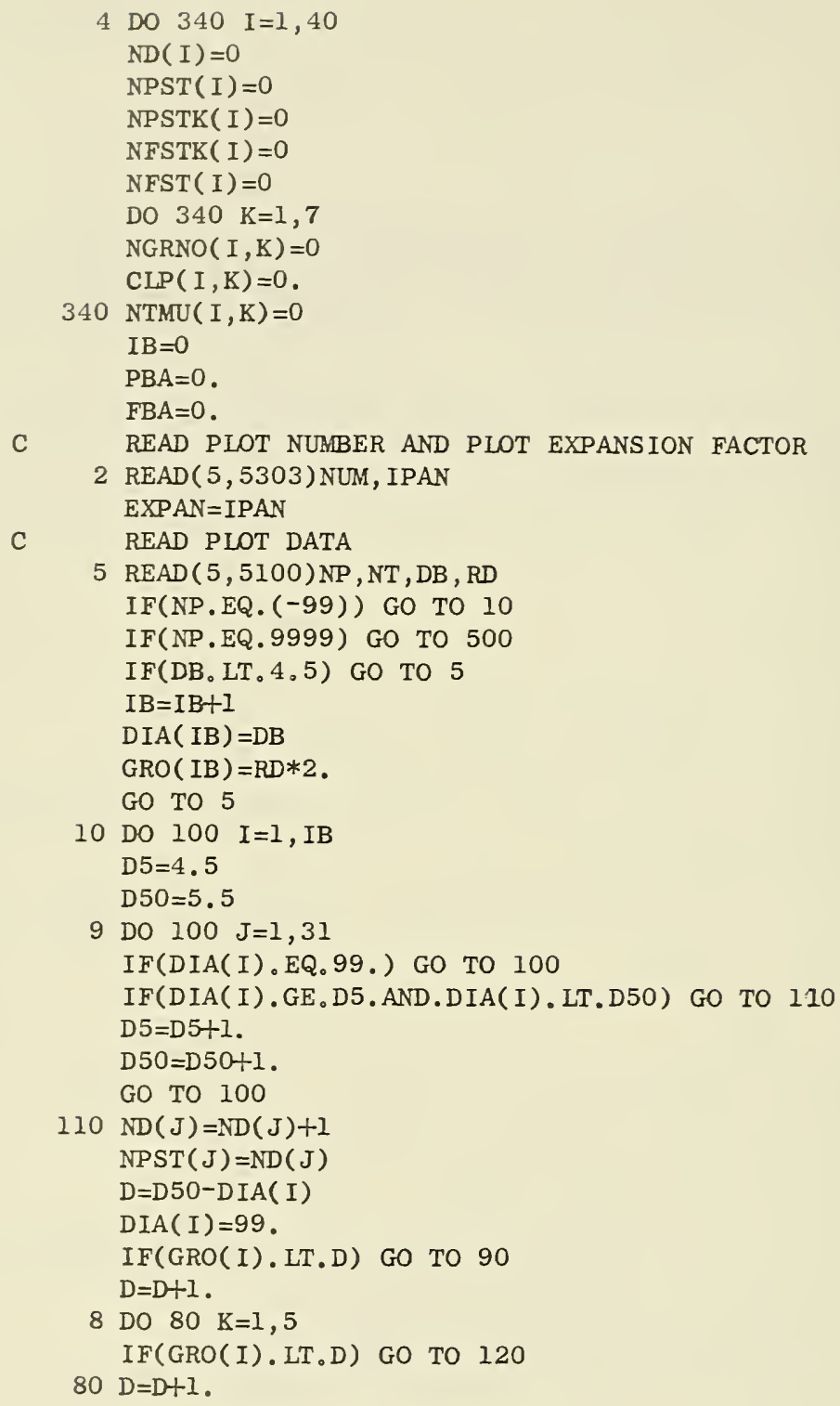


GO TO 100

$90 \operatorname{NGRNO}(\mathrm{J}, 1)=\operatorname{NGRNO}(\mathrm{J}, 1)+1$

GO TO 100

$120 \operatorname{NGRNO}(\mathrm{J}, \mathrm{K}+1)=\operatorname{NGRNO}(\mathrm{J}, \mathrm{K}+1)+1$

100 CONTINUE

20 DO $200 \mathrm{~J}=1,31$

DO $200 \mathrm{~K}=1,6$

$200 \operatorname{CLP}(J, K)=(\operatorname{FLOAT}(\operatorname{NGRNO}(J, K)) / \operatorname{FLOAT}(\operatorname{ND}(J))) * 100$.

WRITE THE TABLE OF GROWTH CLASS NUMBERS AND PERCENTS

WRITE $(6,6008)$ NUM

WRITE $(6,5998)$

$\operatorname{WR} \operatorname{ITE}(6,5997)$

WRITE $(6,5996)$

WRITE $(6,5995)$

WRITE $(6,5994)$

WRITE $(6,5993)$

DO $210 \quad I=1,31$

$\operatorname{MDIA}(\mathrm{I})=\mathrm{I}+4$

210 WRITE $(6,5992) \operatorname{MDIA}(I), \operatorname{ND}(I),(\operatorname{NGRNO}(I, K), \operatorname{CLP}(I, K) ; K=1,6)$

$\mathrm{C}$

CALCULATE THE NUMBER OF TREES MOVING UP BY GROWTH CLASSES

DO $250 \quad I=1,31$

DO $250 \mathrm{~K}=1,6$

$250 \operatorname{NTMU}(I, K)=\operatorname{IFIX}(((\operatorname{CIP}(I, K) *(\operatorname{FLOAT}(\operatorname{NPST}(I)))) / 100)+.5$.

CALCULATE FUTURE STAND TABLE

$\operatorname{NFST}(1)=\operatorname{NTMU}(1,1)$

$\operatorname{NFST}(2)=\operatorname{NTMU}(1,2)+\operatorname{NTMU}(2,1)$

$\operatorname{NFST}(3)=\operatorname{NTMU}(1,3)+\operatorname{NTMU}(2,2)+\operatorname{NTMU}(3,1)$

$\operatorname{NFST}(4)=\operatorname{NTMU}(1,4)+\operatorname{NTMU}(2,3)+\operatorname{NTMU}(3,2)+\operatorname{NTMU}(4,1)$

$\operatorname{NFST}(5)=\operatorname{NTMU}(1,5)+\operatorname{NTMU}(2,4)+\operatorname{NTMU}(3,3)+\operatorname{NTMU}(4,2)+\operatorname{NTMU}(5,1)$

DO $260 \quad I=1,35$

$260 \operatorname{NFST}(I)=\operatorname{NTMU}(I-5,6)+\operatorname{NTMU}(I-4,5)+\operatorname{NTMU}(I-3,4)+\operatorname{NTMU}(I-2,3)+\operatorname{NTMU}(I-2,2$

1) $+\operatorname{NTMU}(I, 1)$

$\operatorname{NFST}(36)=\operatorname{NTMU}(31,6)+\operatorname{NTMU}(32,5)+\operatorname{NTMU}(33,4)+\operatorname{NTMU}(34,3)+\operatorname{NTMU}(35,2)$

$\operatorname{NFST}(37)=\operatorname{NTMU}(32,6)+\operatorname{NTMU}(33,5)+\operatorname{NTMU}(34,4)+\operatorname{NTMU}(35,3)$

$\operatorname{NFST}(38)=\operatorname{NTMU}(33,6)+\operatorname{NTMU}(34,5)+\operatorname{NTMU}(35,4)$

$\operatorname{NFST}(39)=\operatorname{NTMU}(34,6)+\operatorname{NTMU}(35,5)$

$\operatorname{NFST}(40)=\operatorname{NTMU}(35,6)$

DO $270 \quad I=1,36$

$270 \operatorname{MDIA}(\mathrm{I})=\mathrm{I}+4$

WRITE $(6,4000)$

WRITE $(6,4001)$

WRITE $(6,4002)$

WRITE $(6,4003)$ 
DO $275 \mathrm{I}=1,36$

275 WRITE $(6,4004) \operatorname{MDIA}(I), \operatorname{NPST}(I),(\operatorname{NTMU}(I, K), K=1,6), \operatorname{NFST}(I)$

CALCULATE PLOT AND ACRE GROWTH

NTOPST $=0$

DO $280 \quad I=1,31$

280 NTOPST $=$ NTOPST + NPST (I)

NTOFST $=0$

NTPSTK $=0$

NTFSTK $=0$

DO $290 \quad I=1,36$

NTOFST $=$ NTOFST+NFST $(I)$

$\operatorname{NPSTK}(I)=\operatorname{IFIX}((\operatorname{FLOAT}(\operatorname{NPST}(I))) * \operatorname{VOL}(I))$

$\operatorname{NFSTK}(I)=\operatorname{IFIX}((\operatorname{FLOAT}(\operatorname{NFST}(I))) * \operatorname{VOL}(I))$

NTPSTK $=$ NTPSTK + NPSTK $(I)$

290 NTFSTK=NTFSTK+NFSTK(I)

WR ITE $(6,4005)$ NTOPST , NTOFST

NTOTGR=NTFSTK-NTPSTK

$N T P G R=N T O T G R * I P A N$

GRPCT $=($ FLOAT $($ NTOTGR $) / F L O A T($ NTPSTK $)) * 100$.

$\operatorname{WRITE}(6,6000)$

$\operatorname{WRITE}(6,6001)$

$\operatorname{WRITE}(6,6002)$

WRITE $(6,6003)$

DO $335 \mathrm{I}=1,36$

335 WRITE $(6,6004) \mathrm{MDIA}(\mathrm{I}), \operatorname{NPST}(I), \operatorname{NPSTK}(I), \operatorname{NFST}(I), \operatorname{NFSTK}(I)$

C

CALCULATE PRESENT AND FUTURE BASAL AREA

DO $350 \quad I=1,36$

$\mathrm{PBA}=\operatorname{PBA}+((\operatorname{FLOAT}(\operatorname{NPST}(I))) *(((\operatorname{FLOAT}(\operatorname{MDIA}(I))) * * 2) * 0.005454))$

$350 \mathrm{FBA}=\mathrm{FBA}+((\operatorname{FLOAT}(\operatorname{NFST}(I))) *(((\operatorname{FLOAT}(\operatorname{MDIA}(I))) * * 2) * 0.005454))$

$\mathrm{PBAC}=\mathrm{PBA} * \mathrm{EXPAN}$

FBAC $=$ FBA $*$ EXPAN

$B A G=F B A C-P B A C$

WRITE $(6,6005)$ NTPSTK, NTFSTK

WRITE $(6,6006)$ NTOTGR

WRITE $(6,6009)$ NTPGR

WRITE $(6,6007)$ GRPCT

WRITE $(6,6010)$ PBAC

WRITE $(6,6011)$ FBAC

WRITE $(6,6012)$ BAG

GO TO 4

500 STOP

END 
Figure 1

Flow chart for the Fortran IV Program

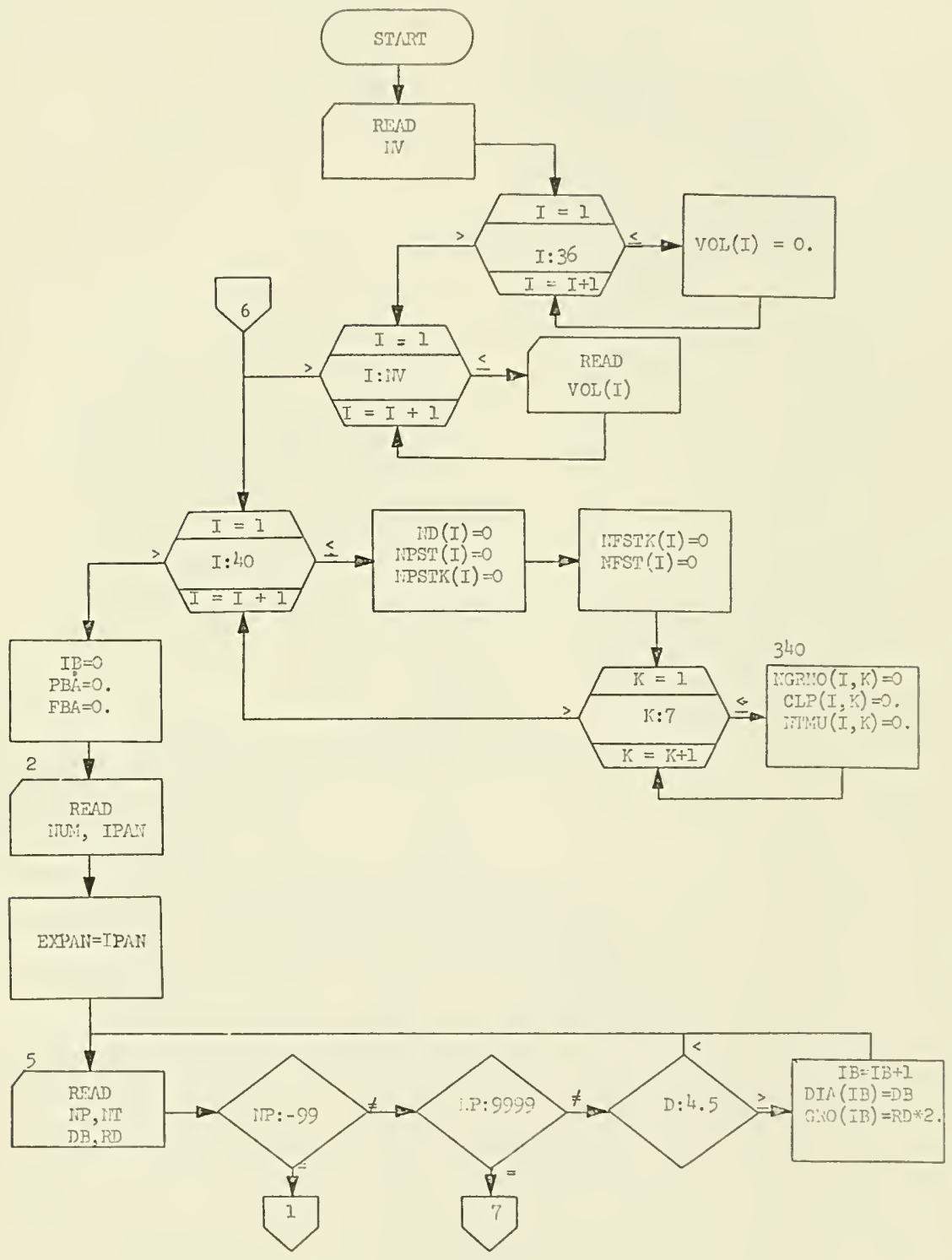




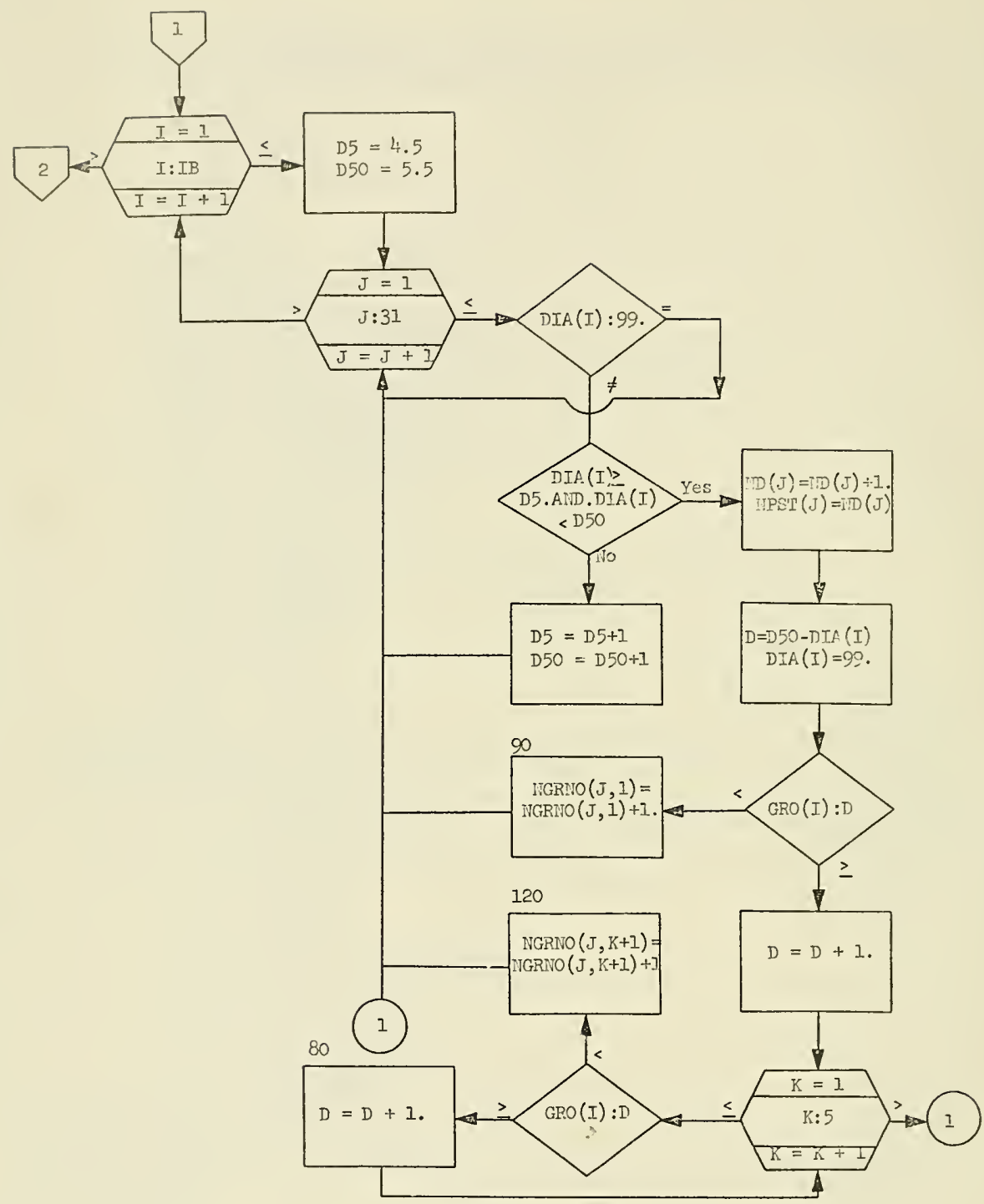




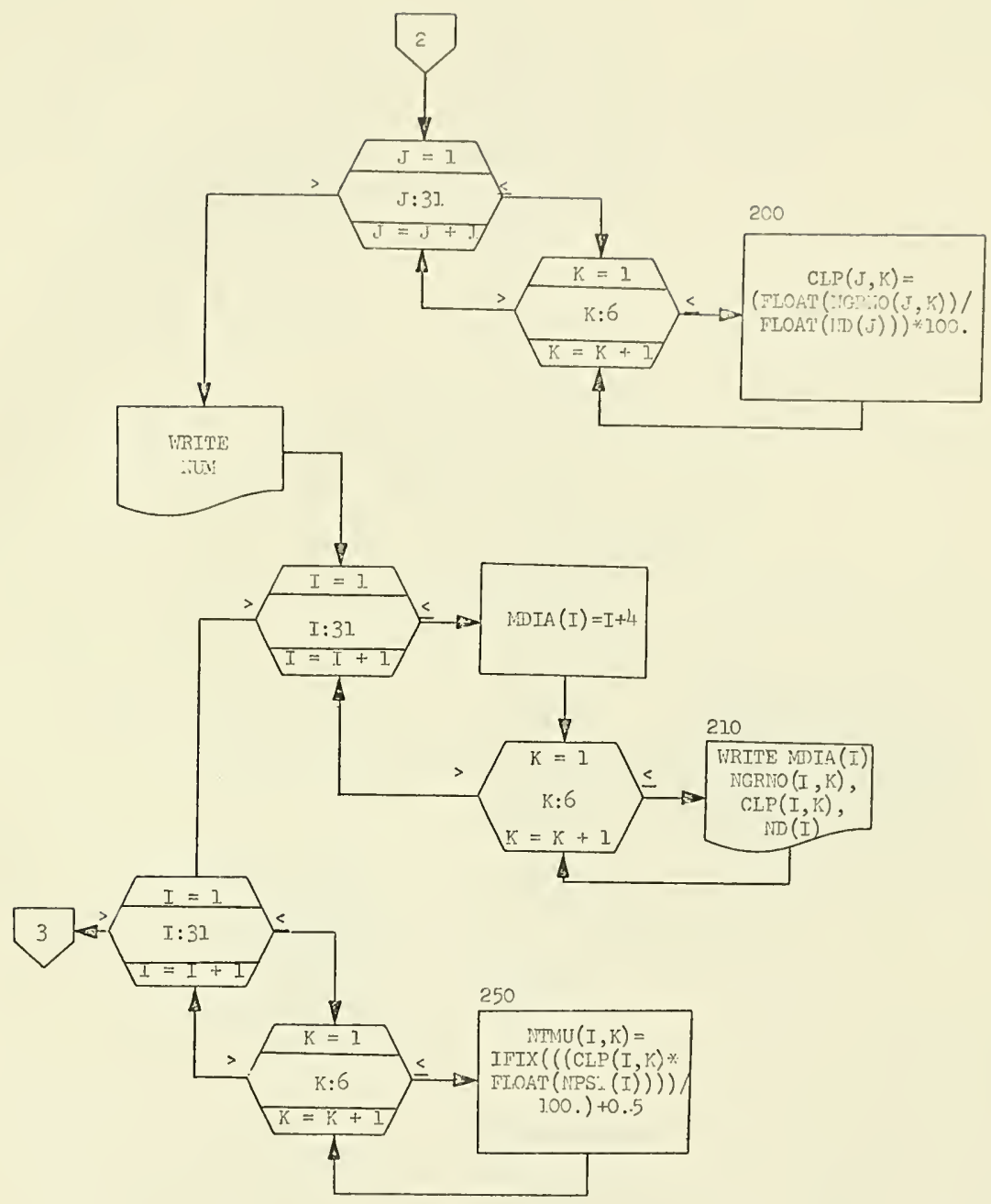




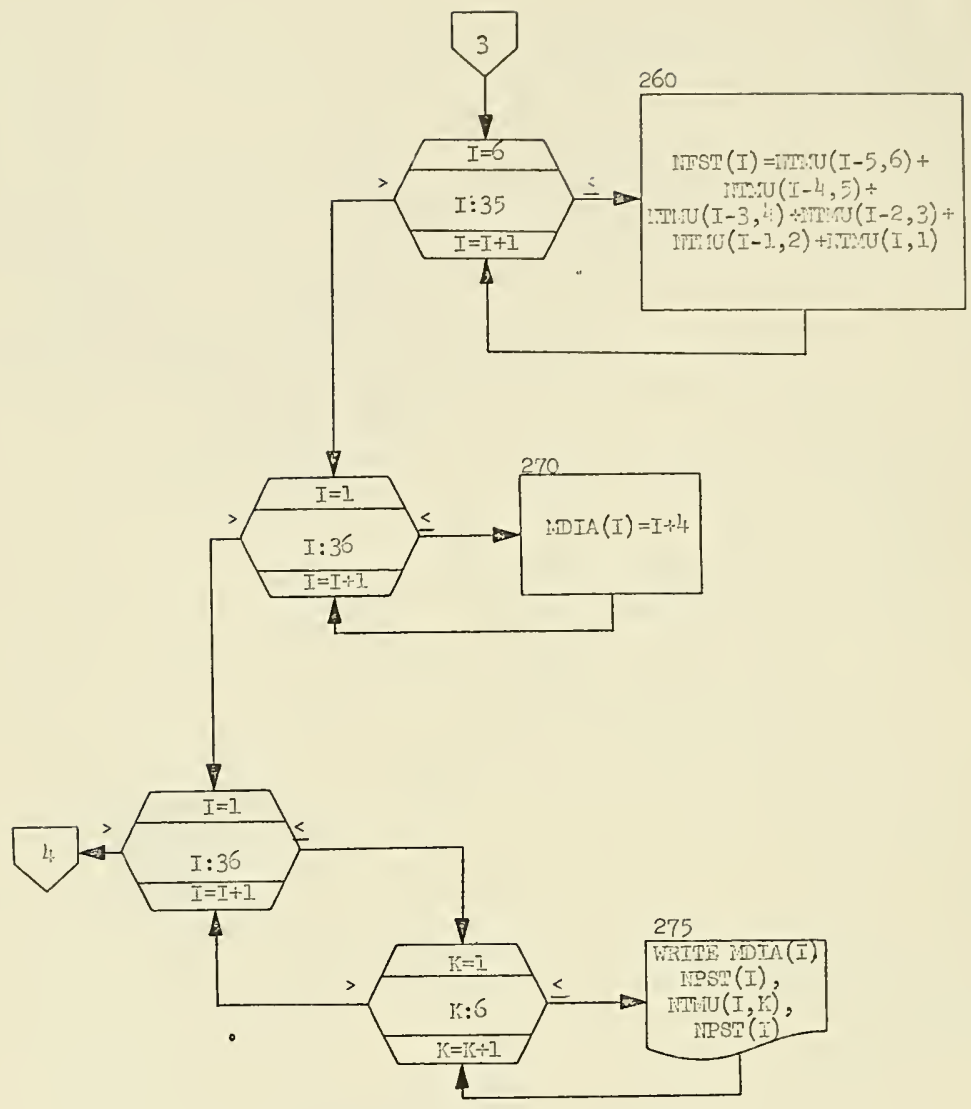




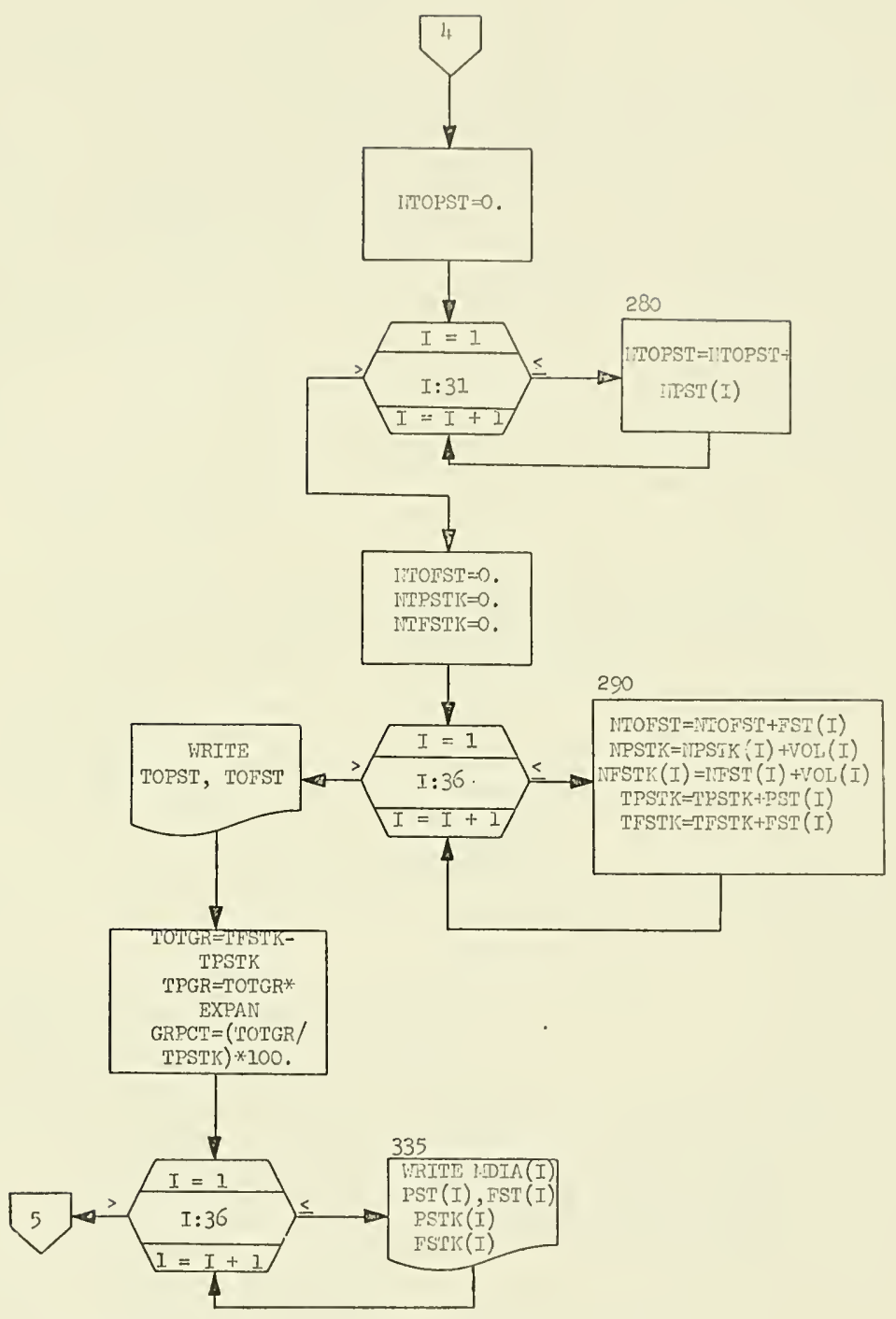




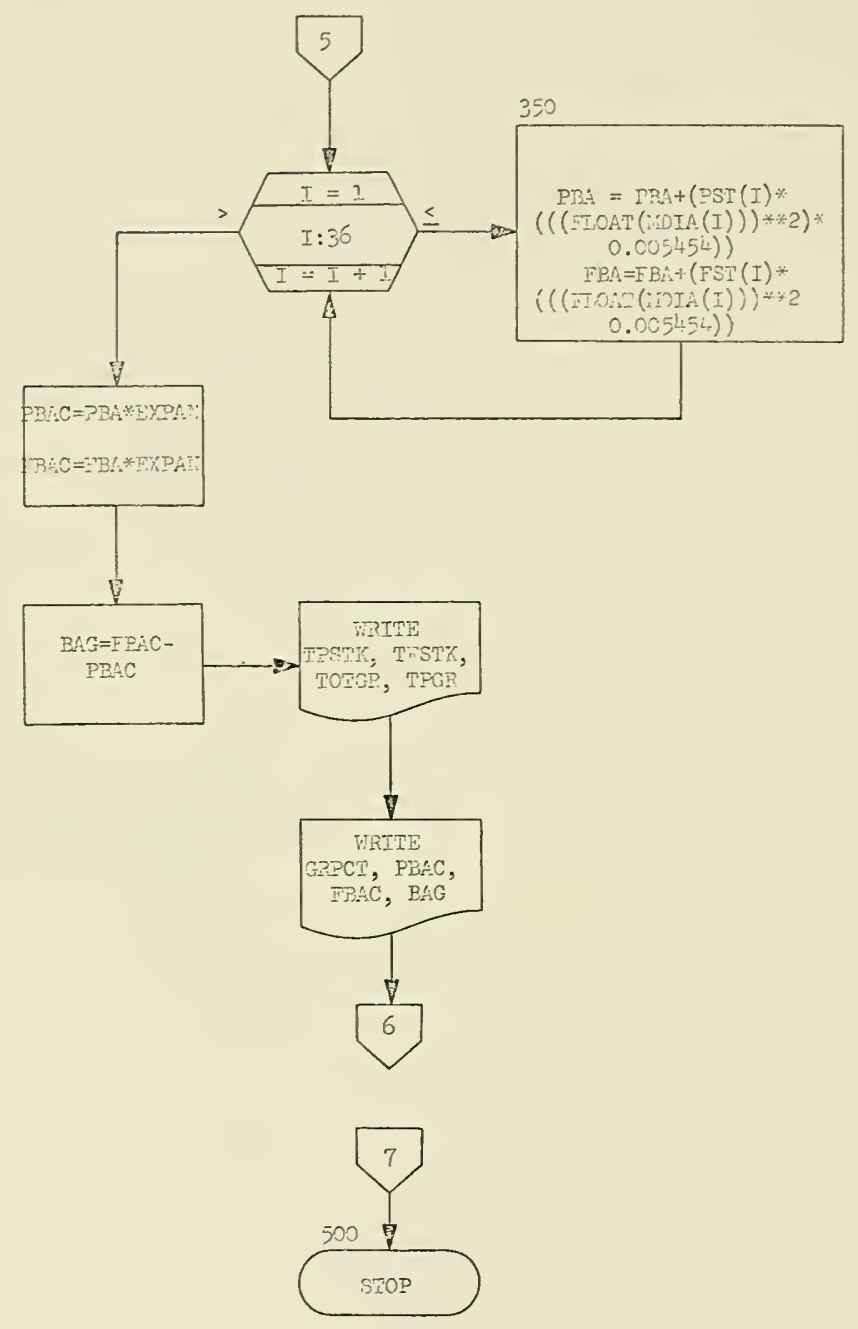




\section{TABLE 2}

Definition of Variables for the Fortran IV Program

VOL $(\mathrm{I})=$ the volume per tree by one-inch diameter classes, of five inches to 40 inches

$\mathrm{ND}(\mathrm{I})=$ the number of trees occuring in each diameter class

NPST $(I)=$ the present stand table for any one plot

$\operatorname{NPSTK}(\mathrm{I})=$ the present stock table for the plot

$\operatorname{NFSTK}(\mathrm{I})=$ the future stock table

$\operatorname{NFST}(\mathrm{I})=$ the future stand table

NGRNO $(I, K)=$ the number of trees on any one growth class, for each diameter class

$\operatorname{CLP}(\mathrm{I}, \mathrm{K})=$ the per cent of the present stand table that occurs in each growth class

$\operatorname{NTMU}(\mathrm{I}, \mathrm{K})=$ the number of trees changing diameter classes

$\mathrm{PBA}=$ present basal area

$\mathrm{FBA}=$ future basal area

NUM $=$ plot number

IPAN $=$ plot expansion factor expressed as an integer

EXPAN = floating point plot expansion factor

$\mathrm{NP}=$ plot number

$\mathrm{NT}=$ tree number

$\mathrm{DB}=$ tree diameter

$\mathrm{RD}=$ ten-year radial growth

DIA $(\mathrm{IB})=$ array of plot tree diameters

$\mathrm{GRO}(\mathrm{IB})=$ array of corresponding diameter growths for each tree

IB $=$ total number of trees recorded on each plot

D5 = lower limit of diameter class

D50 = upper limit of diameter class

$\mathrm{D}=$ difference between diameter and upper diameter limit

MDIA $(\mathrm{I})=$ midpoint of diameter class

NTOPST $=$ total number of trees in present stand table

NTOFST $=$ total number of trees in future stand table

NTPSTK $=$ present plot volume

NTFSTK = future plot volume

NTOTGR = ten-year volume increase per plot

NTPGR = volume growth per acre

GRPCT $=$ growth per cent

$\mathrm{PBAC}=$ present basal area per acre

$\mathrm{FBAC}=$ future basal area per acre

$\mathrm{BAG}=$ ten-year basal area growth

$\mathrm{NV}=$ number of classes available in local volume table, beginning at five inches 
TABLE 3

Local Volume Table for Yellow Poplar in West Virginia

\begin{tabular}{cc|cc|cc}
\hline $\begin{array}{c}\text { DBH } \\
\text { Inches }\end{array}$ & $\begin{array}{c}\text { Volume } \\
\text { Cu. Ft. }\end{array}$ & $\begin{array}{c}\text { DBH } \\
\text { Inches }\end{array}$ & $\begin{array}{c}\text { Volume } \\
\text { Cu. Ft. }\end{array}$ & $\begin{array}{c}\text { DBH } \\
\text { Inches }\end{array}$ & $\begin{array}{c}\text { Volume } \\
\text { Cu. Ft. }\end{array}$ \\
\cline { 1 - 2 } 5 & 3.24 & 17 & 51.97 & 29 & 175.53 \\
6 & 4.40 & 18 & 59.41 & 30 & 189.20 \\
7 & 6.12 & 19 & 67.37 & 31 & 203.39 \\
8 & 8.37 & 20 & 75.85 & 32 & 218.10 \\
9 & 11.14 & 21 & 84.85 & 33 & 233.33 \\
10 & 14.43 & 22 & 94.37 & 34 & 249.07 \\
11 & 18.23 & 23 & 104.40 & 35 & 265.01 \\
12 & 22.56 & 24 & 114.96 & 36 & 282.13 \\
13 & 27.40 & 25 & 126.04 & 37 & 299.43 \\
14 & 32.77 & 26 & 137.63 & 38 & 317.26 \\
15 & 38.65 & 27 & 149.74 & 39 & 335.60 \\
16 & 45.05 & 28 & 162.38 & 40 & 354.46 \\
\hline
\end{tabular}

\section{ILLUSTRATION}

Two one-quarter acre plots having 20 trees; seven trees will be used to illustrate the procedure.

Input - Data are punched on cards and entered into the computer as in Table 4:

1. Card number one shows the number $(\mathrm{k})$ of values used from the local volume table. This is entered under FORMAT (I2) and in this case is 36.

2. The volumes from the local volume table are entered with cards 2 to 37 under FORMAT (F6.2).

3. Card number 38 is a "header" card for the first plot. It shows the plot number and expansion factor under FORMAT (I4,I2). The measurement data then follows with one card for each tree. This consists of the plot and tree numbers under FORMAT (I4,I4), and the dbh and radial growth in FORMAT (F5.1,F4.1). This is shown in cards 39 to 58 . Card number 59 is the last entry for that plot and is a "trail" card with —99 under (FORMAT (I4). Succeeding plots follow with their respective "header" and "trail" cards. 
4. The last information to be entered is a single card with 9999 under FORMAT (I4) to signify that all of the data has been entered into the computer. This is placed after the "trail" card for the last plot.

Output - Tables 5 to 7 show the output format for the program for the first plot. Table 5 is an intermediate output showing the present stand table and the tree shift by diameter class. Table 6 sums the trees according to the number of classes moved, and shows the future stand table. Table 7 is the final output with present and future stock and stand tables, with the pertinent data at the bottom. These values may now be combined with other plot values for management purposes.

TABLE 4

Program Example for Two One-Quarter Acre Plots

\begin{tabular}{|c|c|c|c|}
\hline Card & Data & Format & Explanation \\
\hline 1 & 36 & (I2) & \\
\hline 2 & 3.24 & $(\mathrm{~F} 6.2)$ & Vol./dia. class Table III \\
\hline 3 & 4.40 & $(\mathrm{~F} 6.2)$ & Vol./dia. class Table III \\
\hline 37 & 354.46 & $(\mathrm{~F} 6.2)$ & Vol./dia. class Table III \\
\hline 38 & 14 & $(\mathrm{I} 4, \mathrm{I} 2)$ & $\begin{array}{l}\text { Plot number and expan- } \\
\text { sion factor (header } \\
\text { card) }\end{array}$ \\
\hline 39 & $\begin{array}{llll}1 & 1 & 10.4 & 1.6\end{array}$ & $(\mathrm{I} 4, \mathrm{I} 4, \mathrm{~F} 5.1, \mathrm{~F} 4.1)$ & $\begin{array}{l}\text { Plot \& Tree No., dbh, } \\
\text { growth }\end{array}$ \\
\hline 40 & & & \\
\hline 58 & & & \\
\hline 59 & -99 & (I4) & Plot trail card \\
\hline 61 & $\begin{array}{llll}2 & 1 & 24.3 & 0.7\end{array}$ & $(\mathrm{I} 4, \mathrm{I} 4, \mathrm{~F} 5.1, \mathrm{~F} 4.1)$ & $\begin{array}{l}\text { Plot \& Tree No., dbh, } \\
\text { growth }\end{array}$ \\
\hline 68 & -99 & (I4) & Plot trail card \\
\hline 69 & 9999 & (I4) & End of data card \\
\hline
\end{tabular}


TABLE 5

Intermediate Output Data for Fortran IV, Plot One; the Following Data is for Plot 1 Classes Moved in 10 Years

\begin{tabular}{|c|c|c|c|c|c|c|c|c|c|c|c|c|c|}
\hline \multirow{2}{*}{$\frac{\text { Dia }}{5}$} & \multirow{2}{*}{$\begin{array}{c}\begin{array}{c}\text { No. Of } \\
\text { Trees }\end{array} \\
2\end{array}$} & \multicolumn{2}{|c|}{$\begin{array}{c}0 \\
\text { Num. Pcent }\end{array}$} & \multicolumn{2}{|c|}{$\begin{array}{c}1 \\
\text { Num. Pcent }\end{array}$} & \multicolumn{2}{|c|}{$\begin{array}{c}2 \\
\text { Num. Pcent }\end{array}$} & \multicolumn{2}{|c|}{$\begin{array}{c}3 \\
\text { Num. Pcent }\end{array}$} & \multicolumn{2}{|c|}{$\begin{array}{c}4 \\
\text { Num. Pcent }\end{array}$} & \multicolumn{2}{|c|}{$\begin{array}{c}\mathbf{5} \\
\text { Num. Pcent }\end{array}$} \\
\hline & & 0 & 0.0 & 1 & 50.0 & 1 & 50.0 & 0 & 0.0 & 0 & 0.0 & 0 & 0.0 \\
\hline 6 & 0 & 0 & 0.0 & 0 & 0.0 & 0 & 0.0 & 0 & 0.0 & 0 & 0.0 & 0 & 0.0 \\
\hline 7 & 0 & 0 & 0.0 & 0 & 0.0 & 0 & 0.0 & 0 & 0.0 & 0 & 0.0 & 0 & 0.0 \\
\hline 8 & 2 & 1 & 50.0 & 1 & 50.0 & 0 & 0.0 & 0 & 0.0 & 0 & 0.0 & 0 & 0.0 \\
\hline 9 & 0 & 0 & 0.0 & 0 & 0.0 & 0 & 0.0 & 0 & 0.0 & 0 & 0.0 & 0 & 0.0 \\
\hline 10 & 7 & 0 & 0.0 & 0 & 0.0 & 5 & 71.4 & 1 & 14.3 & 1 & 14.3 & 0 & 0.0 \\
\hline 11 & 1 & 0 & 0.0 & 0 & 0.0 & 0 & 0.0 & 1 & 100.0 & 0 & 0.0 & 0 & 0.0 \\
\hline 12 & 1 & 0 & 0.0 & 0 & 0.0 & 1 & 100.0 & 0 & 0.0 & 0 & 0.0 & 0 & 0.0 \\
\hline 13 & 0 & 0 & 0.0 & 0 & 0.0 & 0 & 0.0 & 0 & 0.0 & 0 & 0.0 & 0 & 0.0 \\
\hline 14 & 0 & 0 & 0.0 & 0 & 0.0 & 0 & 0.0 & 0 & 0.0 & 0 & 0.0 & 0 & 0.0 \\
\hline 15 & 0 & 0 & 0.0 & 0 & 0.0 & 0 & 0.0 & 0 & 0.0 & 0 & 0.0 & 0 & 0.0 \\
\hline 16 & 0 & 0 & 0.0 & 0 & 0.0 & 0 & 0.0 & 0 & 0.0 & 0 & 0.0 & 0 & 0.0 \\
\hline 17 & 1 & 0 & 0.0 & 0 & 0.0 & 1 & 100.0 & 0 & 0.0 & 0 & 0.0 & 0 & 0.0 \\
\hline 18 & 0 & 0 & 0.0 & 0 & 0.0 & 0 & 0.0 & 0 & 0.0 & 0 & 0.0 & 0 & 0.0 \\
\hline 19 & 1 & 0 & 0.0 & 1 & 100.0 & 0 & 0.0 & 0 & 0.0 & 0 & 0.0 & 0 & 0.0 \\
\hline 20 & 1 & 0 & 0.0 & 0 & 0.0 & 0 & 0.0 & 1 & 100.0 & 0 & 0.0 & 0 & 0.0 \\
\hline 21 & 0 & 0 & 0.0 & 0 & 0.0 & 0 & 0.0 & 0 & 0.0 & 0 & 0.0 & 0 & 0.0 \\
\hline 22 & 0 & 0 & 0.0 & 0 & 0.0 & 0 & 0.0 & 0 & 0.0 & 0 & 0.0 & 0 & 0.0 \\
\hline 23 & 1 & 0 & 0.0 & 1 & 100.0 & 0 & 0.0 & 0 & 0.0 & 0 & 0.0 & 0 & 0.0 \\
\hline 24 & 0 & 0 & 0.0 & 0 & 0.0 & 0 & 0.0 & 0 & 0.0 & 0 & 0.0 & 0 & 0.0 \\
\hline 25 & 1 & 0 & 0.0 & 0 & 0.0 & 0 & 0.0 & 0 & 0.0 & 1 & 100.0 & 0 & 0.0 \\
\hline 26 & 0 & 0 & 0.0 & 0 & 0.0 & 0 & 0.0 & 0 & 0.0 & 0 & 0.0 & 0 & 0.0 \\
\hline 27 & 0 & 0 & 0.0 & 0 & 0.0 & 0 & 0.0 & 0 & 0.0 & 0 & 0.0 & 0 & 0.0 \\
\hline 28 & 0 & 0 & 0.0 & 0 & 0.0 & 0 & 0.0 & 0 & 0.0 & 0 & 0.0 & 0 & 0.0 \\
\hline 29 & 0 & 0 & 0.0 & 0 & 0.0 & 0 & 0.0 & 0 & 0.0 & 0 & 0.0 & 0 & 0.0 \\
\hline 30 & 1 & 0 & 0.0 & 0 & 0.0 & 0 & 0.0 & 1 & 100.0 & 0 & 0.0 & 0 & 0.0 \\
\hline 31 & 0 & 0 & 0.0 & 0 & 0.0 & 0 & 0.0 & 0 & 0.0 & 0 & 0.0 & 0 & 0.0 \\
\hline 32 & 0 & 0 & 0.0 & 0 & 0.0 & 0 & 0.0 & 0 & 0.0 & 0 & 0.0 & 0 & 0.0 \\
\hline 33 & 0 & 0 & 0.0 & 0 & 0.0 & 0 & 0.0 & 0 & 0.0 & 0 & 0.0 & 0 & 0.0 \\
\hline 34 & 0 & 0 & 0.0 & 0 & 0.0 & 0 & 0.0 & 0 & 0.0 & 0 & 0.0 & 0 & 0.0 \\
\hline 35 & 1 & 0 & 0.0 & 0 & 0.0 & 0 & 0.0 & 0 & 0.0 & 1 & 100.0 & 0 & 0.0 \\
\hline
\end{tabular}


TABLE 6

Intermediate Output Data for Fortran IV, Plot One

\begin{tabular}{|c|c|c|c|c|c|c|c|c|}
\hline \multirow{2}{*}{ DBH } & \multirow{2}{*}{$\begin{array}{l}\text { Present } \\
\text { Stand } \\
\text { Table }\end{array}$} & \multicolumn{5}{|c|}{$\begin{array}{c}\text { Number of Trees Moving Up } \\
\text { Per Growth Class }\end{array}$} & \multirow{2}{*}{\multicolumn{2}{|c|}{$\begin{array}{l}\text { Future } \\
\text { Stand } \\
\text { Table }\end{array}$}} \\
\hline & & 0 & 1 & 2 & 3 & 4 & & \\
\hline 5 & 2 & 0 & 1 & 1 & 0 & 0 & 0 & 0 \\
\hline 6 & 0 & 0 & 0 & 0 & 0 & 0 & 0 & 1 \\
\hline 7 & 0 & 0 & 0 & 0 & 0 & 0 & 0 & 1 \\
\hline 8 & 2 & 1 & 1 & 0 & 0 & 0 & 0 & 1 \\
\hline 9 & 0 & 0 & 0 & 0 & 0 & 0 & 0 & 1 \\
\hline 10 & 7 & 0 & 0 & 5 & 1 & 1 & 0 & 0 \\
\hline 11 & 1 & 0 & 0 & 0 & 1 & 0 & 0 & 0 \\
\hline 12 & 1 & 0 & 0 & 1 & 0 & 0 & 0 & 5 \\
\hline 13 & 0 & 0 & 0 & 0 & 0 & 0 & 0 & 1 \\
\hline 14 & 0 & 0 & 0 & 0 & 0 & 0 & 0 & 3 \\
\hline 15 & 0 & 0 & 0 & 0 & 0 & 0 & 0 & 0 \\
\hline 16 & 0 & 0 & 0 & 0 & 0 & 0 & 0 & 0 \\
\hline 17 & 1 & 0 & 0 & 1 & 0 & 0 & 0 & 0 \\
\hline 18 & 0 & 0 & 0 & 0 & 0 & 0 & 0 & 0 \\
\hline 19 & 1 & 0 & 1 & 0 & 0 & 0 & 0 & 1 \\
\hline 20 & 1 & 0 & 0 & 0 & 1 & 0 & 0 & 1 \\
\hline 21 & 0 & 0 & 0 & 0 & 0 & 0 & 0 & 0 \\
\hline 22 & 0 & 0 & 0 & 0 & 0 & 0 & 0 & 0 \\
\hline 23 & 1 & 0 & 1 & 0 & 0 & 0 & 0 & 1 \\
\hline 24 & 0 & 0 & 0 & 0 & 0 & 0 & 0 & 1 \\
\hline 25 & 1 & 0 & 0 & 0 & 0 & 1 & 0 & 0 \\
\hline 26 & 0 & 0 & 0 & 0 & 0 & 0 & 0 & 0 \\
\hline 27 & 0 & 0 & 0 & 0 & 0 & 0 & 0 & 0 \\
\hline 28 & 0 & 0 & 0 & 0 & 0 & 0 & 0 & 0 \\
\hline 29 & 0 & 0 & 0 & 0 & 0 & 0 & 0 & 1 \\
\hline 30 & 1 & 0 & 0 & 0 & 1 & 0 & 0 & 0 \\
\hline 31 & 0 & 0 & 0 & 0 & 0 & 0 & 0 & 0 \\
\hline 32 & 0 & 0 & 0 & 0 & 0 & 0 & 0 & 0 \\
\hline 33 & 0 & 0 & 0 & 0 & 0 & 0 & 0 & 1 \\
\hline 34 & 0 & 0 & 0 & 0 & 0 & 0 & 0 & 0 \\
\hline 35 & 1 & 0 & 0 & 0 & 0 & 1 & 0 & 0 \\
\hline 36 & 0 & 0 & 0 & 0 & 0 & 0 & 0 & 0 \\
\hline 37 & 0 & 0 & 0 & 0 & 0 & 0 & 0 & 0 \\
\hline 38 & 0 & 0 & 0 & 0 & 0 & 0 & 0 & 0 \\
\hline 39 & 0 & 0 & 0 & 0 & 0 & 0 & 0 & 1 \\
\hline 40 & 0 & 0 & 0 & 0 & 0 & 0 & 0 & 0 \\
\hline
\end{tabular}




\section{TABLE 7}

Final Output Data for Fortran IV, Plot One

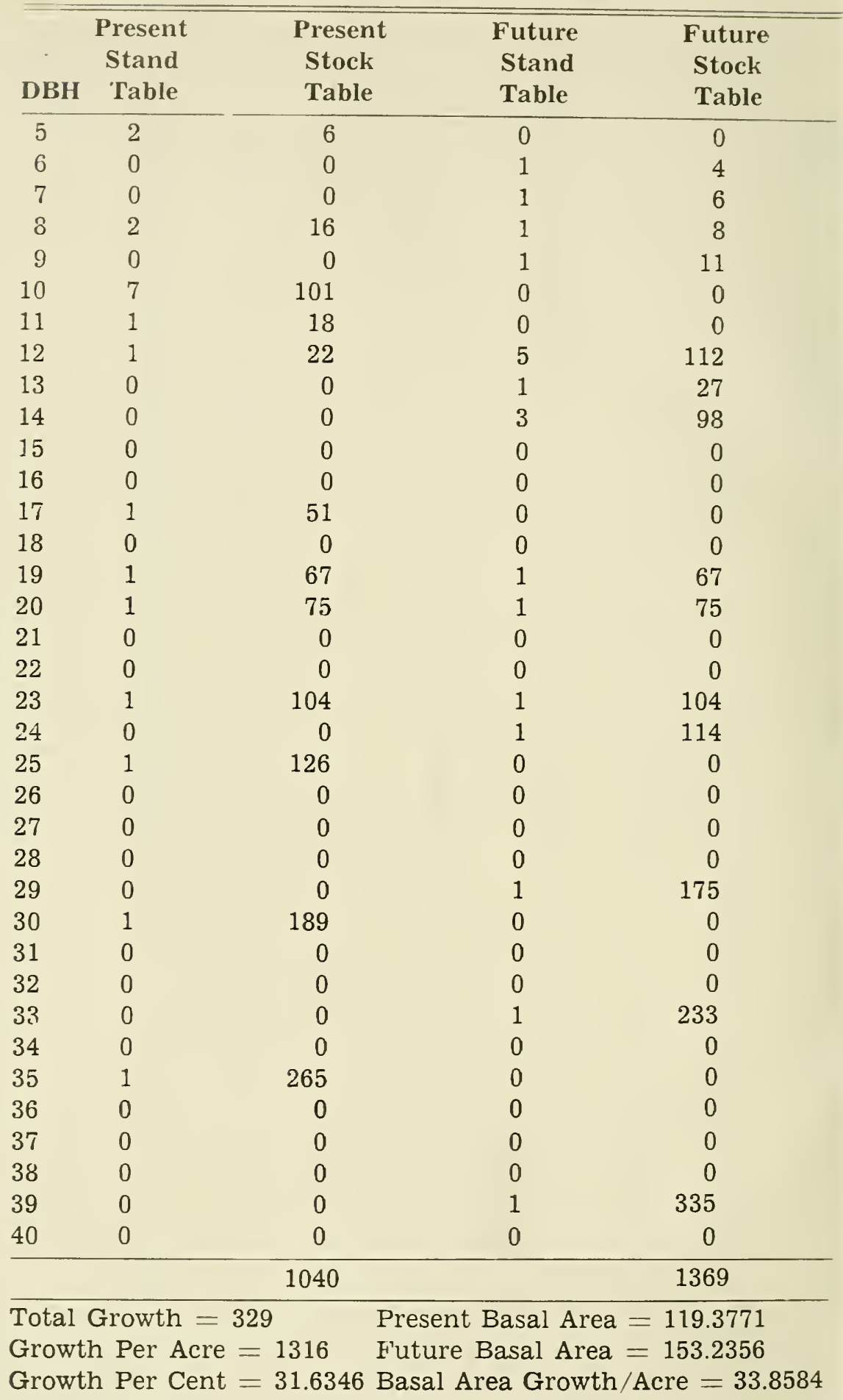





